\title{
Estado de salud y valoración gerontológica en adultos mayores mexicanos ante la pandemia por COVID-19
}

\author{
Eduardo Guzmán-Olea, ${ }^{1 *}$ Raúl A. Agis-Juárez, ${ }^{2}$ Víctor H. Bermúdez-Morales, ${ }^{3}$ Kirvis Torres-Poveda, ${ }^{4}$ \\ Vicente Madrid-Marina, ${ }^{3}$ David López-Romero ${ }^{2}$ y Eloy Maya-Pérez ${ }^{5}$ \\ ${ }^{1}$ Consejo Nacional de Ciencia y Tecnología-Universidad Autónoma del Estado de Hidalgo, Hidalgo; ${ }^{2}$ Universidad Autónoma del Estado de Hidalgo, \\ Instituto de Ciencias de la Salud, Área Académica de Gerontología, Hidalgo; ${ }^{3}$ nstituto Nacional de Salud Pública, Centro de Investigación Sobre \\ Enfermedades Infecciosas, Dirección de Enfermedades Crónicas y Cáncer, Morelos; ${ }^{4}$ Consejo Nacional de Ciencia y Tecnología-Instituto Nacional \\ de Salud Pública, Morelos; ${ }^{5}$ Universidad de Guanajuato, Campus Celaya-Salvatierra, Área Académica de Psicología Clínica, Guanajuato. México
}

\section{Resumen}

Introducción: Los adultos mayores constituyen el grupo más vulnerable ante la pandemia por COVID-19; en México, sus condiciones biopsicosociales podrían potenciar su vulnerabilidad. Método: Se analizó afiliación a sistemas de salud, condiciones de salud y evaluación gerontológica de 3218 adultos mayores conforme a la metodología de la Encuesta Salud, Bienestar y Envejecimiento OPS-México. Resultados: 88.6 \% de los adultos mayores refirió afiliación a un sistema de salud; 30.2, 52.4, 10.3, 4.1 y $5.6 \%$ indicaron padecer diabetes mellitus, hipertensión arterial, enfermedad pulmonar obstructiva crónica, enfermedad cardiaca y evento vascular cerebral, respectivamente; $15.6 \%$ reportó incontinencia urinaria y $11.3 \%$, fecal; $12.1 \%$ de las mujeres indicó haber padecido en algún momento cáncer de mama y $6.3 \%$, cáncer cervicouterino. Se observó hábito de fumar tabaco en $11.1 \%$, riesgo de malnutrición en $32.8 \%$, malnutrición establecida en $4.1 \%$, dependencia funcional para las actividades básicas en $16.3 \%$ e instrumentales de la vida diaria en $17.6 \%$. Conclusión: La evaluación gerontológica integral es fundamental para la atención eficiente de los adultos mayores que padecen COVID-19 y para la adecuada atención por los efectos o condiciones de salud al terminar el confinamiento por la pandemia.

PALABRAS CLAVE: Evaluación gerontológica. Adultos mayores. COVID-19. SARS-CoV-2. México. Vulnerabilidad.

\section{Health status and gerontological evaluation in Mexican older adults in the face of the COVID-19 pandemic}

\begin{abstract}
Introduction: Older adults constitute the most vulnerable population group to the COVID-19 pandemic. In Mexico, their biopsychosocial conditions might intensify their vulnerability. Method: Affiliation to health systems, health conditions and gerontological evaluation of 3,218 older adults were analyzed following the methodology of the PAHO-Mexico Health, Wellbeing and Aging Survey. Results: $88.6 \%$ of older adults referred being affiliated to health systems; $30.2 \%, 52.4 \%, 10.3 \%$, $4.1 \%$ and $5.6 \%$ referred suffering from diabetes mellitus, high blood pressure, chronic obstructive pulmonary disease, heart disease and cerebrovascular disease, respectively; $15.6 \%$ reported urinary incontinence, and $11.3 \%$, fecal incontinence; 12.1 $\%$ of the women referred having suffered from breast cancer at some point, and $6.3 \%$, cervical cancer. The habit of smoking tobacco was observed in $11.1 \%$, risk of malnutrition in $32.8 \%$, established malnutrition in $4.1 \%$, and functional dependence for basic and instrumental activities of daily life in $16.3 \%$ and $17.6 \%$, respectively. Conclusion: Comprehensive gerontological evaluation is essential for efficient care of older adults who suffer from COVID-19, and for adequate care of the effects or health conditions at the conclusion of the confinement imposed by the pandemic.
\end{abstract}

KEY WORDS: Gerontological evaluation. Older adults. COVID-19. SARS-CoV-2. Mexico. Vulnerability.

Correspondencia:

*Eduardo Guzmán-Olea

E-mail: eguzmanol@conacyt.mx

DOI: 10.24875/GMM.20000459
Gac Med Mex. 2020;156:420-425

Disponible en PubMed

www.gacetamedicademexico.com

CC BY-NC-ND (http://creativecommons.org/licenses/by-nc-nd/4.0/). 


\section{Introducción}

A finales de 2019 surgió la enfermedad por coronavirus (COVID-19) en la República Popular China, causada por el coronavirus 2 del síndrome respiratorio agudo severo (SARS-CoV-2); el 11 de marzo de 2020, la Organización Mundial de la Salud decretó el surgimiento de la pandemia. ${ }^{1,2}$ Los reportes iniciales refirieron síndrome febril y síntomas respiratorios como manifestaciones clínicas de la enfermedad; posteriormente se incluyó la actividad inflamatoria pulmonar, trastornos circulatorios, trombosis venosa, síntomas gastrointestinales, hemorragia intracerebral, así como manifestaciones neurológicas y musculares. ${ }^{3-5}$

Por otro lado, debido al riesgo latente de infección a todos los grupos poblacionales, la Organización Mundial de la Salud propuso protocolos generales de acción para el control y contención de la enfermedad; ${ }^{6}$ sin embargo, gracias a los reportes epidemiológicos internacionales se determinó que las personas de 60 años y más, quienes padecen enfermedades crónicas - principalmente hipertensión arterial, diabetes, obesidad, enfermedades renales, respiratorias y cardiacas-, con compromiso inmunológico y los fumadores presentan un riesgo mayor de muerte al padecer COVID-19. ${ }^{7-10}$ En este sentido, debido a que México cuenta con 15.4 millones de adultos mayores (AM) ${ }^{11}$ y que las mayoría de las principales causas de morbilidad y mortalidad en dicho grupo etario son reportadas como factores de riesgo en caso de infección por SARS-CoV-2, constituye un sector altamente vulnerable. $^{12,13}$

Al confirmarse el primer caso de COVID-19 en México, ${ }^{14}$ la Secretaría de Salud instauró medidas generales de contención de la pandemia, pero ante el aumento significativo de los casos se implementaron medidas nacionales que incluyeron la suspensión de actividades educativas, eventos masivos y actividades no esenciales; se estableció la "Jornada Nacional de Sana Distancia", enfocada en promover el distanciamiento social para reducir la frecuencia de contacto entre personas y disminuir el riesgo de propagación de la enfermedad. Al iniciar la fase 2 de la pandemia se estableció la permanencia domiciliaria de la población, con énfasis en la población adulta mayor (60 y más años) y las personas con diagnóstico de hipertensión arterial, diabetes, patologías cardiacas o pulmonares o inmunosupresión. . $^{15-17}$

Debido a que más de la mitad de los AM en México no cuenta con pensión y se ven obligados a trabajar (principalmente en el sector informal), el Estado ha implementado programas de apoyo económico a las personas mayores de 68 años. Para favorecer esta medida, al inicio del confinamiento se proporcionaron por anticipado dos bimestres de ese estímulo económico; sin embargo, aproximadamente $26 \%$ de los AM no dispone de ese apoyo social, por lo que deben trabajar e incumplen con el aislamiento social, con la consecuente exposición a contraer COVID-19. ${ }^{18}$

Posteriormente, a unos días de la finalización de la Jornada Nacional de Sana Distancia por parte del gobierno de México, se registró el mayor número de defunciones (hasta el momento) en 24 horas, lo que ubicó al país como el primer lugar mundial de muertes en las últimas 24 horas causadas por COVID-19; a esa fecha, México había acumulado 101238 enfermos (confirmados), de los cuales $20.38 \%$ correspondió a AM y del total de ellos, $64.2 \%$ requirió hospitalización y falleció $90.04 \%$ de quienes permanecieron hospitalizados. En total hasta ese momento, $29.6 \%$ de todas las defunciones registradas en el país estuvo integrado por AM. ${ }^{19}$

El objetivo del presente trabajo es describir las condiciones de salud y funcionales de los AM mexicanos, antes del inicio de la pandemia causada por SARS-CoV-2, lo cual permitirá ofrecer un panorama general de las condiciones biopsicosociales de la población adulta mayor de México, que apoye a la atención integral durante la pandemia y el posconfinamiento.

\section{Método}

Con un diseño descriptivo transversal se analizó la información obtenida de entrevistas y evaluaciones gerontológicas a 3218 AM originarios de Hidalgo, Puebla, Tlaxcala, Estado de México, Morelos y Ciudad de México, quienes participaron en el Programa Universitario de Atención Gerontológica entre 2018 y 2019. Con la metodología e instrumentos validados para población mexicana de la Encuesta Salud, Bienestar y Envejecimiento (SABE-México), ${ }^{12,20}$ se revisaron los datos sociodemográficos, afiliación, autorreporte de padecer diabetes mellitus, hipertensión arterial, enfermedad pulmonar obstructiva crónica, enfermedades cardiacas, evento cerebral vascular, cáncer e incontinencia fecal y urinaria. Las evaluaciones realizadas abordaron sintomatología depresiva (Escala de Depresión Geriátrica de Yesavage), estado nutricional (escala Mini Nutritional Assessment de Guigoz y Velas), dependencia para la 
realización de actividades básicas de la vida diaria (ABVD, mediante el Índice de Independencia en las Actividades Básicas de la Vida Diaria de Katz), dependencia para la realización de actividades instrumentales de la vida diaria (AIVD, con el Índice de Actividades Instrumentales de la Vida Diaria de Lawton y Brody ${ }^{21} \mathrm{y}$, adicionalmente, el hábito de fumar tabaco.

Las entrevistas y evaluaciones fueron efectuadas por duplas de alumnos del Área Académica de Gerontología de la Universidad Autónoma del Estado de Hidalgo, bajo la supervisión de profesores e investigadores de esta. Los investigadores previamente fueron capacitados con el programa Encuesta SABEMéxico, por la Subdirección de Atención al Envejecimiento del Centro Nacional de Programas Preventivos y Control de Enfermedades (Cenaprece), de la Secretaría de Salud.

Los datos sociodemográficos analizados y sus categorías incluyeron edad (60 a 69, $\geq 70$ años), sexo, residencia (urbana y rural), empleo (trabaja, no trabaja, otro), estado civil (casado y otro) y escolaridad (básica o menor y mayor a la básica). Respecto a las comorbilidades, se consideró la autorreferencia de cada una de ellas por diagnóstico clínico. En cuanto al cáncer, únicamente fueron considerados el de mama y el cervicouterino en las mujeres, así como el de próstata en los hombres; se consideró el padecimiento previo $o$ actual para el registro.

La categorización de las evaluaciones gerontológicas se estableció de la siguiente manera:

- Depresión: sin depresión, depresión leve a moderada y severa.

- Estado nutricional: estado normal, riesgo de malnutrición y malnutrición.

- Dependencia funcional para ABVD y AIVD: sin deterioro, deterioro leve a moderado y deterioro severo.

Todos los análisis e interpretaciones fueron realizados por especialistas de cada una de las áreas. Se llevó a cabo análisis descriptivo y de dispersión de los datos, los cuales se representaron con medias y proporciones, considerando el error estándar y un nivel de significación de $95 \%(p<0.05)$; el procesamiento se realizó mediante el programa estadístico SPSS versión 16 .

El proyecto se llevó a cabo considerando las recomendaciones de la Declaración de Helsinki de 1964 y los lineamientos éticos de la Secretaría de Salud. Al inicio de cada entrevista se informó al AM acerca del Programa Universitario de Atención Gerontológica y se le invitó a participar en él; quienes aceptaron firmaron una carta de consentimiento informado, sin que la negación a participar afectara su atención, evaluación y seguimiento dentro del programa. El proyecto fue revisado y aprobado por la Comisión de Investigación y Ética del Área Académica de Gerontología de la Universidad Autónoma del Estado de Hidalgo.

\section{Resultados}

A partir de 3425 AM se obtuvo una muestra final de 3218 , residentes de áreas urbanas o rurales y edad entre 60 y 105 años al momento del estudio. El programa al que acudieron no depende de ninguna institución de salud. Inicialmente se determinó la afiliación a seguridad social en $88.6 \%$ de los participantes; significativamente se observó mayor predominio de mujeres, residentes de áreas urbanas, personas con empleo y en quienes contaron con escolaridad, esta fue mayor a la básica (Tabla 1).

Respecto al análisis de las principales enfermedades crónicas referidas como factores de riesgo para COVID-19, se identificó diabetes mellitus tipo 2 en $30.2 \%$ de los AM, con significación estadística en las personas de 60 a 69 años, residentes de áreas urbanas, con trabajo, casados y con educación básica o menor. La hipertensión arterial se ubicó como la patología con mayor prevalencia (52.4\%); se observó mayor predominio en hombres, personas con empleo y con nivel educativo básico o menor. La enfermedad pulmonar obstructiva crónica se determinó en $10.3 \%$, mientras que las enfermedades cardiacas únicamente en $4.1 \%$, con mayor predominio en los hombres. Finalmente, $5.6 \%$ indicó haber presentado al menos un evento de enfermedad vascular cerebral (Tabla 2).

En cuanto al cáncer, $12.1 \%$ de las mujeres respondió afirmativamente padecer o haber padecido cáncer de mama, mientras que $6.3 \%$ indicó cáncer cervicouterino; en el caso de los hombres, $11.4 \%$ señaló cáncer de próstata. La incontinencia urinaria fue indicada por $15.6 \%$ de los AM, mientras que $11.3 \%$ refirió incontinencia fecal (Tabla 3 ).

Los resultados de las evaluaciones gerontológicas permitieron evidenciar sintomatología depresiva en $26.5 \%$ del total: $19.7 \%$ con nivel de leve a moderado y $6.8 \%$, nivel severo. Respecto al estado nutricional, $32.8 \%$ presentó riesgo de malnutrición y $4.1 \%$, malnutrición establecida. Para la dependencia funcional a las ABVD (p. e. higiene personal y aseo, vestido, alimentación, movilidad funcional, etcétera), $12.9 \%$ presentó nivel leve a moderado, mientras que $3.4 \%$ 
Tabla 1. Características sociodemográficas de adultos mayores mexicanos participantes del Programa Universitario de Atención Gerontológica

\begin{tabular}{|c|c|c|c|c|c|c|c|c|c|c|c|c|}
\hline \multirow[t]{2}{*}{ Característica } & \multicolumn{2}{|c|}{ Edad (años) } & \multicolumn{2}{|c|}{ Sexo } & \multicolumn{2}{|c|}{ Residencia } & \multicolumn{2}{|c|}{ Empleo } & \multicolumn{2}{|c|}{ Estado civil } & \multicolumn{2}{|c|}{ Escolaridad } \\
\hline & $60-69$ & $\geq 70$ & M & $\mathrm{H}$ & $\mathbf{U}$ & $\mathbf{R}$ & $\mathrm{T}$ & NT & C & 0 & $\leq \mathrm{B}$ & $>\mathrm{B}$ \\
\hline $\begin{array}{l}\text { Población ( } \mathrm{n}=3218,100 \text { \%) } \\
\text { n (\%) }\end{array}$ & $\begin{array}{c}1580 \\
(49.1)\end{array}$ & $\begin{array}{c}1638 \\
(50.9)\end{array}$ & $\begin{array}{l}2204 \\
(68.5)\end{array}$ & $\begin{array}{c}1014 \\
(31.5)\end{array}$ & $\begin{array}{l}2037 \\
(63.3)\end{array}$ & $\begin{array}{c}1181 \\
(36.7)\end{array}$ & $\begin{array}{l}2275 \\
(70.7)\end{array}$ & $\begin{array}{c}943 \\
(29.3)\end{array}$ & $\begin{array}{l}2838 \\
(88.2)\end{array}$ & $\begin{array}{c}380 \\
(11.8)\end{array}$ & $\begin{array}{l}2040 \\
(63.4)\end{array}$ & $\begin{array}{c}1178 \\
(36.6)\end{array}$ \\
\hline $\begin{array}{l}\text { Afiliación ( } n=2851.88 .6 \%) \\
\text { n (\%) }\end{array}$ & $\begin{array}{c}1374 \\
(48.2)\end{array}$ & $\begin{array}{c}1477 \\
(51.8)\end{array}$ & $\begin{array}{l}2061^{\S} \\
(72.3)\end{array}$ & $\begin{array}{c}790 \\
(27.7)\end{array}$ & $\begin{array}{c}1796^{\S} \\
(63)\end{array}$ & $\begin{array}{l}1055 \\
(37)\end{array}$ & $\begin{array}{l}1955^{\S} \\
(68.6)\end{array}$ & $\begin{array}{c}896 \\
(31.4)\end{array}$ & $\begin{array}{l}2589 \\
(90.8)\end{array}$ & $\begin{array}{c}262 \\
(9.2)\end{array}$ & $\begin{array}{c}1702 \\
(59.7)\end{array}$ & $\begin{array}{c}1149 \\
(40.3)\end{array}$ \\
\hline
\end{tabular}

$\mathrm{M}=$ mujeres, $\mathrm{H}=$ hombres, $\mathrm{U}=$ urbano, $\mathrm{R}=$ rural. $\mathrm{T}=$ trabaja, $\mathrm{NT}=$ no trabaja, $\mathrm{C}=$ casado, $\mathrm{O}=$ otros (solteros, viudos), $\leq \mathrm{B}=$ básica (primaria completa) $\mathrm{o}$ menor a la básica;

$>\mathrm{B}=$ mayor a la básica (secundaria, preparatoria, técnico o profesional). De acuerdo con el Instituto Nacional de Estadística y Geografía, se considera población rural cuando tiene menos de 2500 habitantes.

sDiferencia estadísticamente significativa entre los grupos con $p<0.05$

Tabla 2. Principales patologías en 3218 adultos mayores participantes del Programa Universitario de Atención Gerontológica

\begin{tabular}{|c|c|c|c|c|c|c|c|c|c|c|}
\hline \multirow[t]{2}{*}{ Variable } & \multicolumn{2}{|c|}{ DM n = $972(30.2 \%)$} & \multicolumn{2}{|c|}{ HA $n=1686(52.4 \%)$} & \multicolumn{2}{|c|}{$E P O C n=331(10.3 \%)$} & \multicolumn{2}{|c|}{$E C n=132(4.1 \%)$} & \multicolumn{2}{|c|}{ EVC $n=181(5.6 \%)$} \\
\hline & $\mathbf{P}(\%)$ & NP $(\%)$ & $P(\%)$ & NP (\%) & $\mathbf{P}(\%)$ & NP $(\%)$ & $\mathbf{P}(\%)$ & NP $(\%)$ & $\mathbf{P}(\%)$ & NP $(\%)$ \\
\hline $\begin{array}{l}\text { Edad } \\
\begin{array}{l}60-69 \\
\geq 70\end{array}\end{array}$ & $\begin{array}{c}16.8^{\S} \\
13.4\end{array}$ & $\begin{array}{l}32.3 \\
37.5\end{array}$ & $\begin{array}{l}24.9 \\
27.5\end{array}$ & $\begin{array}{l}24.2 \\
23.4\end{array}$ & $\begin{array}{l}4.9 \\
5.4\end{array}$ & $\begin{array}{l}44.2 \\
45.5\end{array}$ & $\begin{array}{l}1.2 \\
2.9\end{array}$ & $\begin{array}{c}47.9 \\
48\end{array}$ & $\begin{array}{l}3.2 \\
2.4\end{array}$ & $\begin{array}{l}45.9 \\
48.5\end{array}$ \\
\hline $\begin{array}{c}\text { Sexo } \\
M \\
H\end{array}$ & $\begin{array}{l}15.9 \\
14.3\end{array}$ & $\begin{array}{l}52.6 \\
17.2\end{array}$ & $\begin{array}{l}23.8 \\
28.6^{\S}\end{array}$ & $\begin{array}{c}44.7 \\
2.9\end{array}$ & $\begin{array}{l}5.2 \\
5.1\end{array}$ & $\begin{array}{l}63.2 \\
26.4\end{array}$ & $\begin{array}{l}1.5 \\
2.6^{\S}\end{array}$ & $\begin{array}{c}67 \\
28.9\end{array}$ & $\begin{array}{c}2.6 \\
3\end{array}$ & $\begin{array}{l}65.9 \\
28.5\end{array}$ \\
\hline $\begin{array}{l}\text { Residencia } \\
\text { U } \\
\text { R }\end{array}$ & $\begin{array}{l}17.3^{\S} \\
12.9\end{array}$ & $\begin{array}{c}46 \\
23.8\end{array}$ & $\begin{array}{l}29.2 \\
23.2\end{array}$ & $\begin{array}{l}34.1 \\
13.5\end{array}$ & $\begin{array}{c}5 \\
5.3\end{array}$ & $\begin{array}{l}58.3 \\
31.4\end{array}$ & $\begin{array}{l}2.3 \\
1.8\end{array}$ & $\begin{array}{c}61 \\
34.9\end{array}$ & $\begin{array}{l}3.4 \\
2.2\end{array}$ & $\begin{array}{l}59.9 \\
34.5\end{array}$ \\
\hline $\begin{array}{c}\text { Empleo } \\
\text { T } \\
\text { NT }\end{array}$ & $\begin{array}{l}17.9^{\S} \\
12.3\end{array}$ & $\begin{array}{c}52.8^{\S} \\
17\end{array}$ & $\begin{array}{c}31.4 \\
21\end{array}$ & $\begin{array}{c}39.3 \\
8.3\end{array}$ & $\begin{array}{l}6.8 \\
3.5\end{array}$ & $\begin{array}{l}63.9 \\
25.8\end{array}$ & $\begin{array}{l}2.7 \\
1.4\end{array}$ & $\begin{array}{c}68 \\
27.9\end{array}$ & $\begin{array}{c}3.6 \\
2\end{array}$ & $\begin{array}{l}67.1 \\
27.3\end{array}$ \\
\hline $\begin{array}{l}\text { Estado civil } \\
\text { C } \\
0\end{array}$ & $\begin{array}{c}22.6^{\S} \\
7.6\end{array}$ & $\begin{array}{c}65.6 \\
4.2\end{array}$ & $\begin{array}{c}43.5 \\
8.9\end{array}$ & $\begin{array}{c}44.7 \\
2.9\end{array}$ & $\begin{array}{l}6.2 \\
4.1\end{array}$ & $\begin{array}{l}82 \\
7.7\end{array}$ & $\begin{array}{l}2.2 \\
1.9\end{array}$ & $\begin{array}{l}86 \\
9.9\end{array}$ & $\begin{array}{l}2.9 \\
2.7\end{array}$ & $\begin{array}{c}85.3 \\
9.1\end{array}$ \\
\hline $\begin{array}{l}\text { Escolaridad } \\
\leq \mathrm{B} \\
>\mathrm{B}\end{array}$ & $\begin{array}{c}20.8^{\S} \\
9.4\end{array}$ & $\begin{array}{l}42.6^{\S} \\
27.2\end{array}$ & $\begin{array}{l}35.6 \\
16.8\end{array}$ & $\begin{array}{l}27.8 \\
19.8\end{array}$ & $\begin{array}{l}5.5 \\
4.8\end{array}$ & $\begin{array}{l}57.9 \\
31.8\end{array}$ & $\begin{array}{c}2 \\
2.1\end{array}$ & $\begin{array}{l}61.4 \\
34.5\end{array}$ & $\begin{array}{l}3.1 \\
2.5\end{array}$ & $\begin{array}{l}60.3 \\
34.1\end{array}$ \\
\hline
\end{tabular}

$\mathrm{DM}=$ diabetes mellitus, $\mathrm{HA}=$ hipertensión arterial, $\mathrm{EPOC}=$ enfermedad pulmonar obstructiva crónica, $\mathrm{EC}=$ enfermedad cardiaca, $\mathrm{EVC}=$ evento cerebral vascular, $\mathrm{P}=$ presentó, $\mathrm{NP}=$ no presentó, $\mathrm{M}=$ mujer, $\mathrm{H}=$ hombre, $\mathrm{U}=$ urbano, $\mathrm{R}=$ rural, $\mathrm{T}=$ trabaja, $\mathrm{NT}=$ no trabaja, $\mathrm{C}=$ casado, $\mathrm{O}=$ otros (solteros, viudos), $\leq \mathrm{B}=$ básica (primaria completa) o menor a la básica, > $\mathrm{B}=$ mayor a la básica.

sDiferencia estadísticamente significativa entre los grupos con $p<0.05$.

Tabla 3. Padecimiento previo o actual de cáncer, incontinencia urinaria y fecal y hábitos tabáquicos en 3218 adultos mayores

\begin{tabular}{|c|c|c|c|c|c|c|}
\hline \multirow[t]{3}{*}{ Condición } & \multicolumn{3}{|c|}{ Referencia de cáncer } & \multicolumn{2}{|c|}{ Incontinencia } & \multirow[t]{2}{*}{ Tabaquismo } \\
\hline & Mama* & $\mathrm{CaCu}^{*}$ & Próstata** & Urinaria & Fecal & \\
\hline & $\%$ (IC $95 \%)$ & $\%$ (IC $95 \%)$ & $\%$ (IC $95 \%)$ & $\%($ IC $95 \%)$ & $\%$ (IC $95 \%)$ & $\%$ (IC $95 \%)$ \\
\hline Refiere & $12.1(11.6-12.9)$ & $6.3(5.8-7.1)$ & $11.4(10.8-12.2)$ & $15.6(14.9-16.2)$ & $11.3(10.6-12.1)$ & $11.1(10.3-11.8)$ \\
\hline No refiere & $87.9(86.2-88.8)$ & 93.7 (92.9-94.3) & $88.6(87.8-89.4)$ & $84.4(83.7-85.2)$ & $88.7(87.6-89.9)$ & $88.9(87.7-90.2)$ \\
\hline p de la diferencia & 0.00 & 0.00 & 0.00 & 0.00 & 0.00 & 0.03 \\
\hline
\end{tabular}

*Únicamente en mujeres. **Únicamente en hombres. Mujeres, $n=2204$ (68.5\%); hombres, $n=1014$ (31.5\%).

$\mathrm{CaCu}=$ cáncer cervicouterino, IC $95 \%$ = intervalo de confianza de $95 \%$. 
Tabla 4. Evaluación gerontológica de $\mathbf{3 2 1 8}$ adultos mayores

\begin{tabular}{|c|c|c|c|c|c|c|c|c|}
\hline \multirow[t]{3}{*}{ Evaluación } & \multirow{2}{*}{\multicolumn{2}{|c|}{ Sintomatología depresiva \% }} & \multirow{2}{*}{\multicolumn{2}{|c|}{ Estado nutricional \% }} & \multicolumn{4}{|c|}{ Dependencia funcional } \\
\hline & & & & & \multicolumn{2}{|c|}{ ABVD $\%$} & \multicolumn{2}{|c|}{ AIVD $\%$} \\
\hline & $\begin{array}{l}\text { Leve-Mod. } \\
\text { (IC } 95 \% \text { ) }\end{array}$ & $\begin{array}{l}\text { Severa } \\
\text { (IC } 95 \% \text { ) }\end{array}$ & $\begin{array}{c}\text { RMN } \\
\text { (IC } 95 \%)\end{array}$ & $\begin{array}{c}\text { Mn } \\
\text { (IC } 95 \%)\end{array}$ & $\begin{array}{l}\text { Leve-Mod. } \\
\text { (IC } 95 \% \text { ) }\end{array}$ & $\begin{array}{l}\text { Severa } \\
\text { (IC } 95 \%)\end{array}$ & $\begin{array}{l}\text { Leve-Mod. } \\
\text { (IC } 95 \% \text { ) }\end{array}$ & $\begin{array}{l}\text { Severa } \\
\text { (IC } 95 \% \text { ) }\end{array}$ \\
\hline Presenta & $19.7(19.1-20.2)$ & $6.8(5.9-7.4)$ & $32.8(31.2-33.7)$ & $4.1(3.2-5)$ & $12.9(11.8-13.7)$ & $3.4(2.2-4.1)$ & $16.2(15.1-17.3)$ & $1.4(0.8-2.1)$ \\
\hline No presenta & \multicolumn{2}{|c|}{$73.5(72.1-74.3)$} & \multicolumn{2}{|c|}{$63.1(61.9-64.5)$} & \multicolumn{2}{|c|}{$83.7(82.6-84.9)$} & \multicolumn{2}{|c|}{$82.4(80.9-84)$} \\
\hline p de la diferencia & 0.00 & 0.00 & 0.00 & 0.00 & 0.00 & 0.00 & 0.00 & 0.00 \\
\hline
\end{tabular}

dependencia severa. Respecto a la dependencia funcional de las AIVD (uso del teléfono, manejo de medicamentos, realización de tareas domésticas, etcétera), $16.2 \%$ señaló dependencia leve a moderada y $1.4 \%$, dependencia severa (Tabla 4).

\section{Discusión}

Las condiciones biopsicosociales de los AM en México los colocan como uno de los sectores más vulnerables ante COVID-19 en nuestro país. Los evidentes esfuerzos para conocer las condiciones de salud de dicho sector son insuficientes ante la situación actual. Debe realizarse un análisis integral que involucre también las condiciones socioeconómicas y las condiciones de independencia y funcionalidad.

La necesidad de contar con seguridad social en salud no se cubre solo con la afiliación, pues aunque el nivel de cobertura es amplio, las deficiencias en la calidad de la atención, la falta de accesibilidad a las instituciones y a los especialistas, la ausencia de personal sanitario y la suspensión de la medicación para patologías preexistentes (como hipertensión, diabetes, cáncer y enfermedades cardiovasculares) llevan a la búsqueda de otras instituciones públicas o privadas, lo que podría generar mayor exposición al SARS-CoV-2, aumentar el riesgo de contagio y generar gasto de bolsillo, que ante la situación actual podría ser catastrófico para los AM. ${ }^{22-25}$ Dada la complejidad en las condiciones individuales de los AM como la cronicidad de multipatologías, la polifarmacia, la fragilidad física, la dependencia y la vulnerabilidad socioeconómica, es necesaria la atención multidisciplinario en caso de padecer COVID-19.

Los aportes aquí presentados permiten puntualizar que los AM con condiciones incapacitantes o dependencia podrían tener mal pronóstico en su recuperación o un desenlace fatal en caso de infección por SARSCoV-2. En los pacientes con deficiencias nutricionales en quienes sea necesaria la ventilación mecánica, la disfagia posterior representa un elemento fundamental en la rehabilitación y sobrevivencia. ${ }^{26}$ La sintomatología depresiva podría verse afectada por el aislamiento social e, incluso, evolucionar y causar deterioro cognitivo leve e intento de suicidio. ${ }^{27,28} \mathrm{La}$ dependencia funcional en actividades básica e instrumentales de la vida diaria podría afectar el cumplimiento de las medidas de higiene y aislamiento necesarias para prevenir el contagio, así como el estado nutricional, el uso racional de medicamentos, el estado físico y la motricidad.

Finalmente, con todo lo anterior, nuestra mirada se centra en evidenciar las complicaciones biopsicosociales que enfrentan y enfrentarán los AM en tiempos de la pandemia y posCOVID-19. Con base en la evaluación gerontológica realizada se fortalece la idea del rediseño y adaptación del sistema educativo de salud en México a las condiciones actuales, la generación de nuevos perfiles profesionales, la implementación de programas basados en el empoderamiento del autocuidado, la capacitación de cuidadores primarios y el diseño de esquemas de atención fundamentados en la promoción de la autonomía. ${ }^{29-33}$

\section{Conclusiones}

Los AM constituyen el grupo poblacional más vulnerable ante la pandemia por COVID-19, principalmente por procesos propios del envejecimiento (por ejemplo, inflamación crónica e inmunosenescencia). La condición de vulnerabilidad de la población mexicana mayor de 60 años aumenta por sus características biopsicosociales, como la complejidad de sus padecimientos, el desarrollo de condiciones de incapacidad o dependencia y la vulnerabilidad económica 
que presentan. Ante las condiciones actuales, resulta fundamental disponer de un diagnóstico integral de los AM, que permita el manejo y la intervención más adecuada en los casos con COVID-19, para atender eficientemente la cronicidad establecida o aumentada de las patologías preexistentes. También será necesario desarrollar estrategias de intervención basadas en la persona, enfocada en los procesos de autocuidado y acciones que permitan la autonomía.

\section{Agradecimientos}

Al Programa Nacional de Atención al Envejecimiento Cenaprece (Secretaría de Salud), por la capacitación para la aplicación de las encuestas y evaluaciones. A los encuestadores y evaluadores del Área Académica de Gerontología, Universidad Autónoma del Estado de Hidalgo.

\section{Conflicto de intereses}

Los autores declaran no tener conflicto de intereses alguno.

\section{Financiamiento}

El proyecto fue financiado por apoyo recurrente de la Universidad Autónoma del Estado de Hidalgo.

\section{Responsabilidades éticas}

Protección de personas y animales. Los autores declaran que para esta investigación no realizaron experimentos en seres humanos ni en animales.

Confidencialidad de los datos. Los autores declaran que siguieron los protocolos de su centro de trabajo sobre la publicación de datos de terceros.

Derecho a la privacidad y consentimiento informado. Los autores obtuvieron el consentimiento informado de los pacientes o sujetos referidos en el artículo. Este documento obra en poder del autor de correspondencia.

\section{Bibliografía}

1. World Health Organization. Coronavirus disease (COVID-19) pandemic. Suiza: World Health Organization; 2020.

2. Cucinotta D, Vanelli M. WHO declares COVID-19 a pandemic. Acta Biomed. 2020;91:157-160

3. Mao L, Wang M, Chen S, He Q, Chang J, Hong C, et al. Neurological manifestations of hospitalized patients with COVID-19 in Wuhan, China: a retrospective case series study. medRxiv. 2020.

4. Jiménez-Ruiz A, García-Grimshaw M, Ruiz-Sandoval JL. Neurologic manifestations of COVID-19. Gac Med Mex. 2020;156:258.
5. Schmulson M, Dávalos MF, Berumen J. Alerta: los síntomas gastrointestinales podrían ser una manifestación de la COVID-19. Rev Gastroenterol Mex. 2020;85:282-287.

6. World Health Organization. What are the international health regulations and emergency committees? Suiza: World Health Organization; 2019.

7. Zhou F, Yu T, Du R, Fan G, Liu Y, Liu Z, et al. Clinical course and risk factors for mortality of adult inpatients with COVID-19 in Wuhan, China: a retrospective cohort study. Lancet. 2020;395:1054-1062.

8. Huang $\mathrm{C}$, Wang $Y$, Li X, Ren L, Zhao J, Hu Y, et al. Clinical features of patients infected with 2019 novel coronavirus in Wuhan, China. Lancet. 2020;6736:1-10

9. Fang J, Liehua D, Lianging Z, Cai Y, Cheung CW, Xia Z. Review of the clinical characteristics of coronavirus disease 2019 (COVID-19). J Gen Intern Med. 2020;5:1545-1549.

10. Secretaría de Salud [Internet]. México: Nuevo coronavirus 2019 nCoV-Comunicado técnico diario; 2020.

11. Instituto Nacional de Estadística, Geografía e Informática [Internet]. México: Estadísticas a propósito del Día Internacional de las Personas de Edad. Datos nacionales; 2019

12. Secretaría de Salud. Encuesta Salud, bienestar y envejecimiento SABE. México: SSa; 2017.

13. Guzmán-Olea E, López-Romero D, Torres-Poveda K, Madrid-Marina V, Luna-Muñoz J, Pimentel-Pérez BM, et al. Condiciones biopsicosociales de adultos mayores mexicanos con diabetes mellitus. Gac Med Mex. 2017:153:794-799.

14. Secretaría de Salud [Internet]. México: Aviso epidemiológico-2020. Enfermedad COVID-19 por SARS-CoV-2; 2020.

15. Secretaría de Salud [Internet]. México: Sana distancia COVID-19; 2020.

16. Ornelas-Aguirre JM. El nuevo coronavirus que llegó de Oriente: análisis de la epidemia inicial en México. Gac Med Mex. 2020:208-216.

17. Suárez V, Suárez-Quezada M, Oros-Ruiz S, Ronquillo-de Jesús E. Epidemiología de COVID-19 en México: del 27 febrero al 30 de abril de 2020. Rev Clin Esp. 2020.

18. Secretaría de Bienestar [Internet]. México: Programa para el Bienestar de las Personas Adultas Mayores; 2019.

19. Dirección General de Epidemiología [Internet]. México: Covid-19 México. Información general; 2020.

20. Lozano ME, Calleja J, Mena R, et al. Investigación clinicoepidemiológica en envejecimiento: metodología del proyecto Encuesta Salud, Bienestar y Envejecimiento (SABE) en México. Rev Soc Peru Med Interna. 2011;24:186-198.

21. Secretaría de Salud. Principios del abordaje gerontológico en la persona adulta mayor e intervenciones básicas. México: Secretaría de Salud/Centro Nacional de Programas Preventivos y Control de Enfermedades; 2012.

22. Guzmán-Olea E, Maya-Pérez E, López-Romero D, Torres-Poveda K, Bermúdez-Morales VH, Madrid Marina V, et al. Cobertura y eficiencia en atención a las principales enfermedades de adultos mayores de Hidalgo, México. Acta Universitaria. 2018;28:33-39

23. Organización Mundial de la Salud [Internet]. Suiza: La COVID-19 afecta significativamente los servicios de salud relacionados con las enfermedades no transmisibles; 2020.

24. Pavón-León P, Reyes-Morales H, Martínez A, Méndez-Maín SM, Gogeascoechea-Trejo MC, Blázquez-Morales MS. Gasto de bolsillo en adultos mayores afiliados a un seguro público de salud en México. Gac Sanit. 2017;31:286-291.

25. Knaul FM, González-Pier E, Gómez-Dantés O, et al. Hacia la cobertura universal en salud: protección social para todos en México. Salud Publica Mex. 2013;55:207-235.

26. Laviano A, Koverech A, Zanetti M. Nutrition support in the time of SARSCoV-2 (COVID-19). Nutrition. 2020;74:110834

27. Lara E, Koyanagi A, Domènech-Abella J, Miret M, Ayuso-Mateos J, Haro JM. The impact of depression on the development of mild cognitive impairment over 3 years of follow-up: a population-based study. Dement Geriatr Cogn Disord. 2017;43:155-169.

28. Conejero I, Olié E, Courtet $\mathrm{P}$, Calati R. Suicide in older adults: current perspectives. Clin Interv Aging. 2018;13:691-699.

29. Ñamendys-Silva S, Domínguez-Cherit G. Recommendations for the management of critically ill adult patients with COVID-19. Gac Med Mex. 2020;156:247-249.

30. Barajas-Ochoa A, Andrade-Romo JS, Ramos-Santillán VO. Retos para la educación médica en México en los tiempos del COVID-19. Gac Med Mex. 2020;156:254-257

31. Guzmán-Olea E, Pimentel-Pérez BM, Salas-Casas A, Armenta-Carrasco Al, Oliver-González LB, Agis-Juárez Raúl A. Prevention of physical dependence and cognitive impairment by implementing an early rehabilitation program in institutionalized elderly. Acta Universitaria. 2016;26:53-59.

32. Guzmán-Olea E, Maya-Pérez E, López-Romero D, Torres-Poveda K, Madrid-Marina V, Pimentel-Pérez BM, et al. Eficacia de un programa de empoderamiento en la capacidad de autocuidado de la salud en adultos mayores mexicanos jubilados. Salud \& Sociedad. 2017:8:10-20.

33. Sánchez-Izquierdo M, Santacreu M, Olmos R, Fernández-Ballesteros R. A training intervention to reduce paternalistic care and promote autonomy: a preliminary study. Clin Interv Aging. 2019;14:1515-1525. 